\title{
Brain perfusion by arterial spin labeling MRI in multiple sclerosis
}

\author{
Tatiana Koudriavtseva ${ }^{1} \cdot$ Domenico Plantone ${ }^{1} \cdot$ Rosaria Renna $^{1} \cdot$ Matilde Inglese $^{2}$
}

Received: 15 March 2015/Revised: 21 May 2015/Accepted: 21 May 2015/Published online: 28 May 2015

(C) Springer-Verlag Berlin Heidelberg 2015

\section{Dear Sirs,}

The role of MRI in multiple sclerosis (MS) has been well established for decades. Moreover, new MRI techniques have been developed to better understand the complex pathogenesis of this disease, since the use of conventional MRI techniques is partially limited by its weak associations with clinical features and low sensitivity for gray matter (GM) involvement and diffuse damage of white matter (WM) [1]. These limitations become even more significant in the disease shift from the predominantly inflammatory to degenerative phase [1].

MS has been traditionally considered a demyelinating inflammatory disorder of the central nervous system; however, vascular involvement and perfusion abnormalities are recently receiving an increasing interest [2]. Earlier PET and SPECT studies showed metabolic alterations and perfusion deficits in cognitively impaired MS patients, particularly at the cortical level in the left frontal and temporal lobes [3]. Recently, a dynamic susceptibility contrast-enhanced (DSC) MRI showed in MS a globally reduced but regionally mixed cerebral blood flow (CBF). Decreased CBF has been demonstrated in both normalappearing WM (NAWM) [4-7] and deep GM [6, 7] in relapsing-remitting MS (RRMS) patients, which had also a significantly reduced $\mathrm{CBF}$ in the putamen compared to patients with clinically isolated syndrome (CIS) [7].

Tatiana Koudriavtseva

tatiana.koud@gmail.com

1 Unit of Neurology, Multiple Sclerosis Center, Regina Elena National Cancer Institute, IFO, Rome, Italy

2 Department of Neurology, Radiology and Neuroscience, Icahn School of Medicine at Mount Sinai, New York, NY, USA
Interestingly, the greater reduction of NAWM CBF was found in primary-progressive MS compared to RRMS [5, 6], though NAWM CBF was decreased even in CIS patients [7]. A regional increase of CBF has been detected in early lesion stages, up to 3 weeks prior to brain-blood barrier (BBB) breakdown with subsequent contrast enhancement [8]. Furthermore, NAWM CBF was shown to significantly correlate with clinical disability [5], whereas GM CBF correlated with neuropsychological dysfunctions [6].

Due to recent increasing availability of higher field strength scanners, a new MRI technique called arterial spin labeling (ASL) has been proposed as a useful research tool in several neurological diseases. Interestingly, a reduction of GM CBF measured by ASL was confirmed in all MS patients compared to healthy controls while NAWM CBF has been alternatively found decreased in some studies [9$11]$, or increased in others $[12,13]$. The reason for the increased NAWM CBF in few studies could be the incomplete separation between NAWM and both WM and enhanced lesions due to the relatively coarse resolution of ASL and non-use of exogenous contrast. Most T1-hypointense lesions were concentrated in WM regions with lower CBF, whilst the T2-hyperintense lesions were distributed in WM regions with both higher and lower CBF [11]. The negative correlations between the T2-hyperintense lesion volume and regional CBF have been showed in several brain areas [14]. Moreover, cerebral vasoreactivity (CVR) from normocapnic to hypercapnic CBF was found diminished in MS patients compared to healthy controls indicating an impaired CBF regulation [15]. Since decreased GM CVR correlated positively with GM atrophy and negatively with the lesion volume, it was hypothesized that the impaired CBF regulation may cause neurodegeneration due to an insufficient blood supply [15]. Similar to 
DSC technique, regional CBF by ASL has been correlated with clinical measures in MS patients, for example the left centrum semiovale CBF was found to be associated with performance on the spell out PASAT [16].

Initially, decreased cerebral perfusion was attributed to hypometabolism secondary to axonal degeneration, however, the study of both perfusion and diffusion tensor imaging supported the concept of primary ischemia in MS [17], as well as no relationship has been found between reduced $\mathrm{CBF}$ and impaired axonal mitochondrial metabolism or astrocytic phosphocreatine metabolism [10]. Hence, the decreased cerebral perfusion could be attributed to inflammatory-related changes according to fluid dynamics. It is known that the NAWM and GM manifest a constant, low-grade inflammation, which could lead to a venular vasodilation with slowing, reduced perfusion. The active lesions, coincident with BBB breakdown even in their early stage, determine a local fluid leakage with increased perfusion. Such lesions are less frequent in the deep GM; thus, it is difficult to see increased perfusion here. The inflammation together with perfusion changes can cause neuronal dysfunction even in absence of degeneration. This might explain the link between perfusion and clinical symptoms.

Therefore, perfusion MRI could provide a new potential outcome measures especially in progressive MS characterized by a more severe decrease in CBF and by a lower accrual of both Gd-enhanced or new/enlarged T2-weighted lesions. Moreover, perfusion MRI might represent a suitable tool to investigate microcirculation abnormalities and provide a more comprehensive measure of inflammation in RRMS.

Although ASL technique has limitations such as the relatively low spatial resolution and the requirement of a careful position of region of interests to prevent partial volume effects with lesions and blood vessels, it provides a sensitive measure of cerebral perfusion without the need of a contrast since it uses the blood as an endogenous contrast agent [13]. Therefore, ASL might become a useful tool to evaluate experimental neuroprotective drugs in proof-ofconcept studies and reduce both patients' exposure to gadolinium and the costs of clinical trials requesting serial MRIs.

Conflicts of interest No conflict of interest or financial interest is reported. T. Koudriavtseva reports consulting fees from Bayer Schering, and Institutional grant from Merck Serono, Biogen Idec, Novartis, Bayer Schering outside the submitted work. M. Inglese reports research grant support from NIH (R56 NS079116-01A1), NMSS (RG 4916A2/1 and RG 5120-A-3) and Novartis Pharmaceuticals outside the submitted work. Other authors have nothing to declare.

Ethical standard All human studies have been approved by the appropriate ethics committee and have therefore been performed in accordance with the ethical standards laid down in the 1964 Declaration of Helsinki and its later amendments.

\section{References}

1. Bakshi R, Thompson AJ, Rocca MA, Pelletier D, Dousset V, Barkhof F, Inglese M, Guttmann CR, Horsfield MA, Filippi M (2008) MRI in multiple sclerosis: current status and future prospects. Lancet Neurol 7(7):615-625. doi:10.1016/S14744422(08)70137-6

2. D'haeseleer M, Cambron M, Vanopdenbosch L, De Keyser J (2011) Vascular aspects of multiple sclerosis. Lancet Neurol 10:657-666. doi:10.1016/S1474-4422(11)70105-3

3. Pozzilli C, Passafiume D, Anzini A, Borsellino G, Koudriavtseva T, Sarlo G, Fieschi C (1992) Cognitive and brain imaging measures of multiple sclerosis. Ital J Neurol Sci 13(9 Suppl 14):133-136

4. Law M, Saindane AM, Ge Y, Babb JS, Johnson G, Mannon LJ, Herbert J, Grossman RI (2004) Microvascular abnormality in relapsing-remitting multiple sclerosis: perfusion MR imaging findings in normal-appearing white matter. Radiology 231(3):645-652

5. Adhya S, Johnson G, Herbert J, Jaggi H, Babb JS, Grossman RI, Inglese M (2006) Pattern of hemodynamic impairment in multiple sclerosis: dynamic susceptibility contrast perfusion MR imaging at 3.0 T. Neuroimage 33(4):1029-1035

6. Inglese M, Park SJ, Johnson G, Babb JS, Miles L, Jaggi H, Herbert J, Grossman RI (2007) Deep gray matter perfusion in multiple sclerosis: dynamic susceptibility contrast perfusion magnetic resonance imaging at 3 T. Arch Neurol 64(2):196-202

7. Varga AW, Johnson G, Babb JS, Herbert J, Grossman RI, Inglese M (2009) White matter hemodynamic abnormalities precede subcortical gray matter changes in multiple sclerosis. J Neurol Sci 282(1-2):28-33. doi:10.1016/j.jns.2008.12.036

8. Wuerfel J, Bellmann-Strobl J, Brunecker P, Aktas O, McFarland H, Villringer A, Zipp F (2004) Changes in cerebral perfusion precede plaque formation in multiple sclerosis: a longitudinal perfusion MRI study. Brain 127(Pt 1):111-119

9. D'haeseleer M, Beelen R, Fierens Y, Cambron M, Vanbinst AM, Verborgh C, Demey J, De Keyser J (2013) Cerebral hypoperfusion in multiple sclerosis is reversible and mediated by endothelin-1. Proc Natl Acad Sci USA 110(14):5654-5658. doi:10. 1073/pnas. 1222560110

10. Steen C, Dhaeseleer M, Hoogduin JM, Fierens Y, Cambron M, Mostert JP, Heersema DJ, Koch MW, De Keyser J (2013) Cerebral white matter blood flow and energy metabolism in multiple sclerosis. Mult Scler 19(10):1282-1289. doi:10.1177/ 1352458513477228

11. Narayana PA, Zhou Y, Hasan KM, Datta S, Sun X, Wolinsky JS (2014) Hypoperfusion and T1-hypointense lesions in white matter in multiple sclerosis. Mult Scler 20(3):365-373. doi:10.1177/ 1352458513495936

12. Rashid W, Parkes LM, Ingle GT, Chard DT, Toosy AT, Altmann DR, Symms MR, Tofts PS, Thompson AJ, Miller DH (2004) Abnormalities of cerebral perfusion in multiple sclerosis. J Neurol Neurosurg Psychiatry 75(9):1288-1293

13. Paling D, Thade Petersen E, Tozer DJ, Altmann DR, WheelerKingshott CA, Kapoor R, Miller DH, Golay X (2014) Cerebral arterial bolus arrival time is prolonged in multiple sclerosis and associated with disability. J Cereb Blood Flow Metab 34(1):34-42

14. Ota M, Sato N, Nakata Y, Ito K, Kamiya K, Maikusa N, Ogawa M, Okamoto T, Obu S, Noda T, Araki M, Yamamura T, Kunugi $\mathrm{H}$ (2013) Abnormalities of cerebral blood flow in multiple 
sclerosis: a pseudocontinuous arterial spin labeling MRI study. Magn Reson Imaging 31(6):990-995. doi:10.1016/j.mri.2013.03. 016

15. Marshall O, Lu H, Brisset JC, Xu F, Liu P, Herbert J, Grossman RI, Ge Y (2014) Impaired cerebrovascular reactivity in multiple sclerosis. JAMA Neurol 71:1275-1281

16. D'haeseleer M, Steen C, Hoogduin JM, van Osch MJ, Fierens Y, Cambron M, Koch MW, De Keyser J (2013) Performance on paced auditory serial addition test and cerebral blood flow in multiple sclerosis. Acta Neurol Scand 128(5):e26-e29

17. Saindane AM, Law M, Ge Y, Johnson G, Babb JS, Grossman RI (2007) Correlation of diffusion tensor and dynamic perfusion MR imaging metrics in normal-appearing corpus callosum: support for primary hypoperfusion in multiple sclerosis. AJNR Am J Neuroradiol 28(4):767-772 